\title{
Almost periodicity of some error terms in prime number theory
}

by

Jerzy Kaczorowski (Poznań) and Olivier Ramaré (Lille)

1. Introduction and statement of results. The aim of this paper is to investigate distribution of values of a large class of functions of arithmetic significance assuming a suitably generalized Riemann Hypothesis. Probably the simplest example of a member of this class is defined by the following formula:

(1) $\phi_{0}(v)= \begin{cases}e^{-v / 2}\left(-\psi_{0}\left(e^{v}\right)+e^{v}-\frac{1}{2} \log \left(1-e^{-2 v}\right)-\log 2 \pi\right) & \text { if } v>0, \\ e^{-v / 2}\left(\widetilde{\psi}_{0}\left(e^{-v}\right)+e^{v}+v+\frac{1}{2} \log \frac{1-e^{v}}{1+e^{v}}+C\right) & \text { if } v<0,\end{cases}$

where $C$ is the Euler constant and as usual (for $x>1$ )

$$
\begin{gathered}
\psi(x)=\sum_{n \leq x} \Lambda(n), \quad \widetilde{\psi}(x)=\sum_{n \leq x} \Lambda(n) / n, \\
\psi_{0}(x)=\frac{1}{2}(\psi(x+0)+\psi(x-0)), \quad \widetilde{\psi}_{0}(x)=\frac{1}{2}(\widetilde{\psi}(x+0)+\widetilde{\psi}(x-0)) .
\end{gathered}
$$

This function for positive $v$ is only a mild modification of the normalized remainder term in the prime number formula, where we take the effect of the trivial zeros of the zeta function into account. The proper definition of $\phi_{0}$ for negative values of the argument follows from the work of the first named author [8] and essentially comes from the functional equation of the Riemann zeta function. For real $y$ let

$$
N_{y}(R)=\frac{1}{R} \int_{-\infty}^{\log R} \mathbb{1}_{\left\{\phi_{0}(v)>y\right\}} e^{v} d v .
$$

Our problem is to see how this number and related quantities behave. Though no real number is known for which $\pi(x)>\operatorname{li} x$, the first named author succeeded in [9] in proving under the Riemann Hypothesis that the

2000 Mathematics Subject Classification: Primary 11N05; Secondary 11M26, 11K70, $42 \mathrm{~A} 75$.

J. Kaczorowski partially supported by the KBN Grant number 2 PO3A 02417. 
set of such $x$ has a positive asymptotic lower density, which in our setting is translated into $\liminf \operatorname{in}_{R \rightarrow \infty} N_{-1}(R)>0$. Even better, he showed that for some constant $c_{0}>1$, every interval of the shape $\left[V, c_{0} V\right], V>1$, contains a positive proportion of such points and the same holds when -1 is replaced by any real number $y$. In fact the limsup and liminf of $N_{y}(R)$ as $R$ goes to infinity are $>0$ and $<1$.

Similar problems related to the distribution of primes in arithmetic progressions are of interest. The reader is referred to the survey paper [11] and the literature cited there. The common feature of these results is that they depend on a kind of almost periodicity (cf. also [13]). It is also clear that the method used in the proofs is of a general character and can successfully be applied to many similar problems. The principal aim of this paper is to consider the whole subject from a general point of view. It seems that the framework of the Selberg class is appropriate here.

Let $s=\sigma+i$ and $\bar{f}(s):=\overline{f(\bar{s})}$. The Selberg class $\mathcal{S}$ (cf. [14]) is defined by the following axioms:

(i) (Dirichlet series) Every $F \in \mathcal{S}$ is a Dirichlet series

$$
F(s)=\sum_{n=1}^{\infty} a(n) n^{-s},
$$

absolutely convergent for $\sigma>1$.

(ii) (Analytic continuation) There exists an integer $m \geq 0$ such that $(s-1)^{m} F(s)$ is entire of finite order.

(iii) (Functional equation) $F \in \mathcal{S}$ satisfies a functional equation of type $\Phi(s)=\omega \bar{\Phi}(1-s)$, where

$$
\Phi(s)=Q^{s} \prod_{j=1}^{r} \Gamma\left(\lambda_{j} s+\mu_{j}\right) F(s)=\gamma(s) F(s),
$$

say, with $Q>0, \lambda_{j}>0, \operatorname{Re} \mu_{j} \geq 0$ and $|\omega|=1$.

(iv) (Ramanujan hypothesis) For every $\varepsilon>0$, $a(n) \ll n^{\varepsilon}$.

(v) (Euler product) $F \in \mathcal{S}$ satisfies $\log F(s)=\sum_{n=1}^{\infty} b(n) n^{-s}$, where $b(n)=0$ unless $n=p^{k}$ with $k \geq 1$, and $b(n) \ll n^{\theta}$ for some $\theta<1 / 2$.

The Selberg class contains most $L$-functions used in number theory. The most obvious examples are the Riemann zeta function and the shifts $L(s+i \theta), \theta \in \mathbb{R}$, of Dirichlet $L$-functions with primitive character $\chi(\bmod q)$, $q \geq 2$. Other examples include Dedekind zeta functions of algebraic number fields, and Hecke $L$-functions formed with primitive characters. Moreover, the Artin $L$-functions $L(s, \varrho, K / \mathbb{Q})$ associated with irreducible representations of the Galois group $\operatorname{Gal}(K / \mathbb{Q})$ belong to $\mathcal{S}$ provided a standard conjecture holds. The $L$-functions $L_{f}(s)$ associated with holomorphic newforms 
$f(z)$ on congruence subgroups of $\mathrm{SL}(2, \mathbb{Z})$ belong to $\mathcal{S}$ once suitably normalized. The same is true for the non-holomorphic ones, provided certain conjectures hold. The Rankin-Selberg convolution of two normalized $L$-functions associated with holomorphic newforms is in $\mathcal{S}$, and the same is true for the symmetric square $L$-function associated with a holomorphic newform on $\mathrm{SL}(2, \mathbb{Z})$. Finally, we remark that many other important $L$-functions would belong to $\mathcal{S}$, provided certain well known conjectures, such as the Langlands conjecture, hold.

We refer to [12] for basic facts concerning $\mathcal{S}$. The minimal integer $m$ in (ii) is called the polar order of $F$ and denoted by $m_{F}$. We remark that the function $\gamma(s)$ in (iii) is defined uniquely up to a multiplicative constant. We call it the $\gamma$-factor of $F$ and denote by $\gamma_{F}$. It is known that $F \in \mathcal{S}$ has trivial zeros at points

$$
-\frac{\mu_{j}+k}{\lambda_{j}}, \quad 1 \leq j \leq r, k \geq 0 .
$$

Because of a possible pole at $s=1$, the trivial zero at $s=0$, if it exists, has multiplicity

$$
\#\left\{1 \leq j \leq r: \mu_{j}=0\right\}-m_{F} .
$$

All other zeros are called non-trivial and lie in the vertical strip $0 \leq \sigma \leq 1$. We expect that all non-trivial zeros lie on the critical line $\sigma=1 / 2$. In other words we expect that the Generalized Riemann Hypothesis (GRH) holds in the Selberg class.

Let $F \in \mathcal{S}$ and let $\varrho=\beta+i \gamma$ denote the generic non-trivial zero of $F$. Moreover, let $\omega$ denote the generic trivial zero of $F$. For a complex number $z$ from the upper half-plane $(\operatorname{Im} z>0)$ we write

$$
k(z, F):=\sum_{\operatorname{Im} \varrho>0} e^{\varrho z}
$$

and for $\operatorname{Re} z>0$ let

$$
k\left(z, \gamma_{F}\right):=\sum_{\omega} e^{\omega z}
$$

It is easy to verify that both series converge in the indicated half-planes, the convergence being uniform on compact subsets. Hence $k(\cdot, F)$ is holomorphic for $\operatorname{Im} z>0$, and $k\left(\cdot, \gamma_{F}\right)$ for $\operatorname{Re} z>0$.

Moreover, we define

$$
K(z, F):=\int_{i \infty}^{z} k(s, F) d s,
$$

where the integration is taken along the vertical half-line. For $\operatorname{Im} z>0$ we 
have $K(z, F)=\sum_{\operatorname{Im} \varrho>0} e^{\varrho z} / \varrho$. For real $x \neq 0$ let

$$
\begin{aligned}
& K\left(x, \gamma_{F}\right):= \begin{cases}\int_{1}^{x} k\left(t, \gamma_{F}\right) d t & \text { if } x>0, \\
\int_{-1}^{x} e^{t} k\left(-t, \gamma_{F}\right) d t & \text { if } x<0,\end{cases} \\
& f(x, F):=\lim _{y \rightarrow 0^{+}}(K(x+i y, F)+\overline{K(x+i y, \bar{F})}) \text {, } \\
& \mathcal{F}(x, F):=e^{-x / 2} f(x, F) .
\end{aligned}
$$

We also write

$$
\Lambda_{F}(n):=b(n) \log n, \quad \psi(x, F):=\sum_{n \leq x} \Lambda_{F}(n), \quad \widetilde{\psi}(x, F):=\sum_{n \leq x} \frac{\Lambda_{F}(n)}{n},
$$

so that according to (v) we have, for $\sigma>1$,

$$
\frac{F^{\prime}}{F}(s)=-\sum_{n=1}^{\infty} \frac{\Lambda_{F}(n)}{n^{s}} .
$$

Theorem 1. (a) (Analytic continuation of $k\left(z, \gamma_{F}\right)$ ) The following formula gives meromorphic continuation of $k\left(z, \gamma_{F}\right)$ to the whole complex plane:

$$
k\left(z, \gamma_{F}\right)=\sum_{j=1}^{r} \frac{e^{-\mu_{j} z / \lambda_{j}}}{1-e^{-z / \lambda_{j}}}-m_{F}
$$

(b) (Analytic continuation of $k(z, F)) k(z, F)$ has meromorphic continuation to the Riemann surface $M$ of $\log z$. For $z \in M$ we have

$$
k(z, F)=\frac{1}{2 \pi i}\left(k\left(z, \gamma_{F}\right)-e^{z} k\left(-z, \bar{\gamma}_{F}\right)+\left(1-e^{z}\right) m_{F}\right) \log z+N_{1}(z, F),
$$

where $N_{1}$ is single-valued and meromorphic on $\mathbb{C}$ having simple poles at most at the points $z=0$ or $z= \pm \log n$ for a positive integer $n$. We have

$$
\operatorname{Res}_{z=\log n} N_{1}(z, F)=\frac{1}{2 \pi i} \Lambda_{F}(n), \quad \operatorname{Res}_{z=-\log n} N_{1}(z, F)=\frac{1}{2 \pi i} \cdot \frac{\bar{\Lambda}_{F}(n)}{n} .
$$

(c) (Functional equation) Write points $z \in M$ in the form $z=r e^{i a}$, $r>0, a \in \mathbb{R}$ and let $M \ni z \mapsto z^{c} \in M$ be defined by $\left(r e^{i a}\right)^{c}=r e^{-i a}$. Then for all $z \in M$ we have

$$
k(z, F)+\overline{k\left(z^{c}, \bar{F}\right)}=m_{F} e^{z}-k\left(z, \gamma_{F}\right)-\sum_{\gamma=0} e^{\beta z},
$$

where the summation is taken over all non-trivial, real zeros of $F$ (if any). 
(d) (Boundary values) For $x>0, x \neq \log n$ we have

$$
f(x, F)=-\psi\left(e^{x}, F\right)+m_{F} e^{x}-K\left(x, \gamma_{F}\right)-\sum_{\operatorname{Im} \varrho=0} \frac{e^{\beta x}}{\beta}+c_{F},
$$

whereas for $x<0, x \neq-\log n$ we have

$$
f(x, F)=-\widetilde{\psi}\left(e^{|x|}, \bar{F}\right)+m_{F} x-K\left(x, \gamma_{F}\right)-\sum_{\operatorname{Im} \varrho=0} \frac{e^{\beta x}}{\beta}+d_{F},
$$

where $c_{F}$ and $d_{F}$ are constants depending on $F$.

(e) (Almost periodicity) Under $G R H$ the function $\mathcal{F}(\cdot, F)$ is almost periodic in the $L^{2}$-sense of Stepanov (see Section 4 below for the definition).

Let $\mathcal{X}_{\mathcal{S}}$ denote the $\mathbb{C}$-subspace spanned by functions $\mathcal{F}(\cdot, F), F \in \mathcal{S}$, and $\overline{\mathcal{X}}_{\mathcal{S}}$ its closure in the space of almost periodic functions in the $L^{2}$ sense of Stepanov. Due to the fact that the Selberg class contains a lot of zeta-functions used in number theory, the class $\overline{\mathcal{X}}_{\mathcal{S}}$ is sufficiently large to study many interesting arithmetic problems. Aside from the distribution of primes in arithmetic progressions we can treat prime ideals in ideal classes, Chebotarev type questions, distribution of Fourier coefficients of newforms and many others. In most cases the smaller class $\mathcal{X}_{\mathcal{S}}$ is already sufficient. For example in [11] the following $\phi$-function has been used extensively:

$$
\phi(x, a, q)=\frac{1}{\varphi(q)} \sum_{\chi(\bmod q)} \overline{\chi(a)} \mathcal{F}\left(x, L\left(s, \chi^{\prime}\right)\right)
$$

where $q \geq 1,(a, q)=1$ and $\chi^{\prime}$ denotes the primitive Dirichlet character induced by $\chi$. Here is a less standard example. Let

$$
E: \quad y^{2}=x^{3}+a x+b, \quad a, b \in \mathbb{Z},
$$

be an elliptic curve. According to the Shimura-Taniyama-Weil conjecture, proved in an important special case by A. Wiles and R. Taylor (cf. [18], [16]) and then in full generality in [5], the associated Hasse-Weil $L$-function $L_{E}$ is generated by a modular form of weight 2 . Hence $\widetilde{L}_{E}(s)=L_{E}(s+1 / 2)$ belongs to the Selberg class and the corresponding $\mathcal{F}$-function is in $\mathcal{X}_{\mathcal{S}}$. Our study thus applies to the distribution of the numbers $a_{E}(p)=\# E\left(\mathbb{F}_{p}\right)$ when $p$ runs over primes.

For a real-valued $\phi \in \overline{\mathcal{X}}_{\mathcal{S}}$ let us write

$$
N_{y, \phi}(R)=\frac{1}{R} \int_{-\infty}^{\log R} \mathbb{1}_{\{\phi(v)>y\}} e^{v} d v=\nabla_{y, \phi}(\log R) .
$$

Looking at numerical data for particular $\phi \in \mathcal{X}_{\mathcal{S}}$ (cf. e.g. [10]) together with some theoretical insight, it seems unlikely that $N_{y, \phi}(R)$ should have a 
limit at infinity. This quantity will most probably oscillate. We make this precise in the following conjecture.

CONJECTURE. For every real-valued function $\phi \in \overline{\mathcal{X}}_{\mathcal{S}}$ and every real number $y$, there exists an almost periodic function in the $L^{2}$-sense of Stepanov, say $\Delta_{y, \phi}$, such that

$$
N_{y, \phi}(R) \sim \Delta_{y, \phi}(\log R) \quad(R \rightarrow \infty) .
$$

THEOREM 2. Let $f$ be a real-valued almost periodic function in the $L^{2}$ sense of Stepanov. Then there exists a countable set $\mathcal{Y}$ such that for real $y \notin \mathcal{Y}$, the function $\mathbb{1}_{\{f(t)>y\}}$ is almost periodic in the $L^{2}$-sense of Weyl. In particular assuming $G R H$, for every real-valued $\phi \in \overline{\mathcal{X}}_{\mathcal{S}}$ and every real number $y$ outside an at most countable set $\mathcal{Y}_{\phi}$, the corresponding density $\nabla_{y, \phi}$ is a 1-Lipschitz almost periodic function in the $L^{2}$-sense of Weyl.

We now introduce a whole bunch of densities. Let $w$ be a non-negative non-decreasing $L^{2}$-function on $\mathbb{R}$ whose restriction to $(-\infty, 0]$ is in $L^{1}$, and such that $W(T)=\int_{-\infty}^{T} w(t) d t \rightarrow \infty$ as $T$ goes to infinity. We also impose that $w \in C^{1}(\mathbb{R})$ except maybe at finitely many points. We call such a function admissible. For real-valued $\phi \in \overline{\mathcal{X}}_{\mathcal{S}}$ we then define the relevant density by

$$
N_{y, \phi}(R, w)=\frac{1}{W(\log R)} \int_{-\infty}^{\log R} \mathbb{1}_{\{\phi(v)>y\}} w(v) d v .
$$

TheOREm 3. Assume GRH. Then for every real-valued $\phi \in \overline{\mathcal{X}}_{\mathcal{S}}$ there exists an at most countable set $\mathcal{Y}_{\phi}$ such that for any $y$ in $\mathbb{R} \backslash \mathcal{Y}_{\phi}$ we can find $a \delta_{y}$ such that for all admissible functions $w$ for which $w(t)=o(W(t))$ as $t$ goes to infinity, we have

$$
N_{y, \phi}(R, w) \rightarrow \delta_{y} \quad(R \rightarrow \infty) .
$$

Thus, for instance, for almost all $y \in \mathbb{R}$, the expression

$$
\frac{a+1}{\log ^{a+1} R} \sum_{r \leq R, \psi(r)-r>y \sqrt{r}} \frac{\log ^{a} r}{r} \quad(a \geq 0)
$$

has a limit as $R$ goes to infinity. This theorem would be a consequence of the aforementioned conjecture, and note that it just misses the conjecture. It is also remarkable that there should (conjecturally) be such a change of behaviour between the case $w(t)=o(W(t))$ and the case $w(t)=W(t)$. The set $\mathcal{Y}$ in question is in fact a subset of the set of points where $M\left(\mathbb{1}_{\left\{\phi_{0}(t)>y\right\}}\right)$ (cf. (15)) does exist. Recalling a theorem of Jessen [7], this value is also the derivative of the convex function $M\left(\left(\phi_{0}(t)-y\right)^{+}\right)$and these two quantities exist simultaneously. 
Almost periodic functions in the sense of Bohr or Stepanov have the feature that their behaviour at infinity can be deduced from that in finite time, a feature which is not shared by almost periodic functions in the sense of Weyl or Besicovitch. We can however prove that $\nabla_{y, \phi}$ has this property for every real-valued $\phi \in \mathcal{X}_{\mathcal{S}}$ and under GRH. Let $\widehat{\mathbb{R}}(\phi)$ denote the Bohr compactification relative to $\phi$ (cf. Section 4 for a definition). We readily see that, for $v \in \mathbb{R}$, we have $\nabla_{y, \phi}(v)=\nabla_{y, \phi_{v}}(0)$. Here, and in the following, $\phi_{v}$ or in general $f_{v}$ for any function $f$ denotes the shift of argument by $v$ : $f_{v}(x):=f(x+v)$. Since $\nabla_{y, \phi_{\tau}}(0)$ is well defined for $\tau \in \widehat{\mathbb{R}}(\phi)$, we thus have an extension of $\nabla_{y, \phi}(\cdot)$ to $\widehat{\mathbb{R}}(\phi)$.

THEOREM 4. Let $y$ be a real number and assume GRH. Then for every real-valued $\phi \in \mathcal{X}_{\mathcal{S}}$ the corresponding density $\nabla_{y, \phi}(\cdot)$ is an upper semicontinuous function over $\widehat{\mathbb{R}}(\phi)$. For all real $y$ with at most two exceptions (depending on $\phi) \nabla_{y, \phi}(\cdot)$ is $d_{\phi}$-continuous on $\mathbb{R}$.

In many concrete cases it is easy to show that exceptional values of $y$ do not exist. This is for example the case for $\phi_{0}$ in (1).

Writing Theorem 4 with $N_{y, \phi}$ instead of $\nabla_{y, \phi}$, noticing that $\nabla_{y, \phi}(\cdot)_{v}=$ $\nabla_{y, \phi_{v}}(\cdot)$ and using only real variables, we get (cf. Section 4 for the definition of $\left.\|\cdot\|_{S^{2}}\right)$

$$
\begin{aligned}
& \forall v \in \mathbb{R}, \quad \forall \varepsilon>0, \exists \alpha>0, \forall v^{\prime} \in \mathbb{R}, \\
& \quad\left\|\phi_{v}-\phi_{v^{\prime}}\right\|_{S^{2}} \leq \alpha \Rightarrow\left|N_{y, \phi}\left(e^{v}\right)-N_{y, \phi}\left(e^{v^{\prime}}\right)\right| \leq \varepsilon .
\end{aligned}
$$

We can prove a more uniform version, namely

$\forall v \in \mathbb{R}, \forall$ bounded interval $I$ of $\mathbb{R}, \forall \varepsilon>0, \exists \alpha>0, \forall v^{\prime} \in \mathbb{R}$,

$$
\left\|\phi_{v}-\phi_{v^{\prime}}\right\|_{S^{2}} \leq \alpha \Rightarrow \max _{t \in I}\left|N_{y, \phi}\left(e^{v+t}\right)-N_{y, \phi}\left(e^{v^{\prime}+t}\right)\right| \leq \varepsilon .
$$

We can thus find again the behaviour of $\phi$ over a compact interval in fairly regularly spaced "windows", the main defect of this result being that these windows may not overlap. Theorem 2 when applicable is better from this viewpoint. Translated into similar terms, it says

$$
\begin{aligned}
& \forall \varepsilon>0, \exists \alpha>0, \exists V>0, \forall\left(v, v^{\prime}\right) \in \mathbb{R}^{2}, \\
& \left\|\phi_{v}-\phi_{v^{\prime}}\right\|_{S^{2}} \leq \alpha \Rightarrow \max _{x \in \mathbb{R}} \int_{x}^{x+V}\left|N_{y, \phi}\left(e^{v}\right)-N_{y, \phi}\left(e^{v^{\prime}}\right)\right|^{2} d t \leq \varepsilon V .
\end{aligned}
$$

2. Proof of Theorem 1. (a) Using (2) and (3) we have, for $\operatorname{Re} z>0$,

$$
k\left(z, \gamma_{F}\right)=\sum_{j=1}^{r} \sum_{k=0}^{\infty} e^{-\frac{\mu_{j}+k}{\lambda_{j}} z}-m_{F}=\sum_{j=1}^{r} \frac{e^{-\mu_{j} z / \lambda_{j}}}{1-e^{-z / \lambda_{j}}}-m_{F},
$$

as required. 
(b) We apply the method used in [8] with some modifications. Let

$$
k_{0}(z, F)=\sum_{\operatorname{Re} \mu_{j}=0, \operatorname{Im} \mu_{j}>0} e^{\mu_{j} z / \lambda_{j}}
$$

and let $\mathcal{C}$ denote the contour consisting of two half-lines $s=-b+i t, \infty>$ $t \geq 0$, and $s=1+b+i t, 0 \leq t<\infty$, where $b$ is positive, and a smooth curve $l$ joining $-b$ and $1+b$ lying on the upper half-plane (except for the end points). If $b$ is sufficiently small and $l$ sufficiently close to the real axis we have

$$
\begin{aligned}
2 \pi i\left(k(z, F)+k_{0}(z, F)\right) & =\int_{\mathcal{C}} \frac{F^{\prime}}{F}(s) e^{s z} d s=\left(\int_{-b+i \infty}^{-b}+\int_{l}+\int_{1+b}^{1+b+i \infty}\right) \frac{F^{\prime}}{F}(s) e^{s z} d s \\
& =k_{1}(z, F)+k_{2}(z, F)+h(z, F),
\end{aligned}
$$

say. $h(z, F)$ poses no problems: it is entire of finite order. Using the functional equation of $F$ we obtain

$$
k_{1}(z, F)=-2 \log Q \frac{1}{z} e^{-b z}-\sum_{j=1}^{r} \lambda_{j} k_{11}(z, j)-\sum_{j=1}^{r} \lambda_{j} k_{12}(z, j)-k_{13}(z, F),
$$

where

$$
\begin{aligned}
k_{11}(z, j) & =\int_{-b+i \infty}^{-b} \frac{\Gamma^{\prime}}{\Gamma}\left(\lambda_{j} s+\mu_{j}\right) e^{s z} d s \\
k_{12}(z, j) & =\int_{-b+i \infty}^{-b} \frac{\Gamma^{\prime}}{\Gamma}\left(\lambda_{j}(1-s)+\bar{\mu}_{j}\right) e^{s z} d s \\
k_{13}(z, F) & =\int_{-b+i \infty}^{-b} \frac{\bar{F}^{\prime}}{\bar{F}}(1-s) e^{s z} d s .
\end{aligned}
$$

Let $l_{j}$ denote a smooth path joining $-b+1 / \lambda_{j}$ and $-b$ lying inside the set $\left\{s=\sigma+i t:-b<\sigma<-b+1 / \lambda_{j}, 0<t<1\right\} \cup\{-b\} \cup\left\{-b+1 / \lambda_{j}\right\}$. When $l_{j}$ is sufficiently close to the real axis we have

$$
k_{11}(z, j)=\int_{-b+1 / \lambda_{j}+i \infty}^{-b+1 / \lambda_{j}} \frac{\Gamma^{\prime}}{\Gamma}\left(\lambda_{j} s+\mu_{j}\right) e^{s z} d s+H_{j}(z)+R_{j}(z)
$$

where

$$
H_{j}(z)=\int_{l_{j}} \frac{\Gamma^{\prime}}{\Gamma}\left(\lambda_{j} s+\mu_{j}\right) e^{s z} d s
$$

and

$$
R_{j}(z)= \begin{cases}\frac{2 \pi i}{\lambda_{j}} e^{\mu_{j} z} & \text { if } \operatorname{Re} \mu_{j}=0, \operatorname{Im} \mu_{j}<0 \\ 0 & \text { otherwise }\end{cases}
$$


Changing variable and using the functional equation of the $\Gamma$-function in the form

$$
\frac{\Gamma^{\prime}}{\Gamma}(w+1)=\frac{1}{w}+\frac{\Gamma^{\prime}}{\Gamma}(w)
$$

we obtain

$$
\left(1-e^{z / \lambda_{j}}\right) k_{11}(z, j)=e^{z / \lambda_{j}} I(z)+H_{j}(z)-R_{j}(z),
$$

where

$$
I(z)=\int_{-b+i \infty}^{-b} \frac{e^{s z}}{\lambda_{j} s+\mu_{j}} d s .
$$

Easy computations show that

$$
I(z)=-\frac{1}{\lambda_{j}} e^{-\mu_{j} z / \lambda_{j}} E_{1}\left(\left(b-\frac{\mu_{j}}{\lambda_{j}}\right) z\right)+c_{j} e^{-\mu_{j} z / \lambda_{j}},
$$

where $c_{j}$ is a constant depending on $\lambda_{j}$ and $\mu_{j}$ and

$$
E_{1}(z)=-E i(-z)=-\log z-C-\sum_{n=1}^{\infty} \frac{(-1)^{n} z^{n}}{n ! \cdot n}
$$

( $C$ denotes the Euler constant). This implies

(7) $\quad\left(1-e^{z / \lambda_{j}}\right) k_{11}(z, j)$

$$
=-\frac{1}{\lambda_{j}} e^{\left(1 / \lambda_{j}-\mu_{j} / \lambda_{j}\right) z} E_{1}\left(\left(b-\frac{\mu_{j}}{\lambda_{j}}\right) z\right)+c_{j} e^{\left(1 / \lambda_{j}-\mu_{j} / \lambda_{j}\right) z}+H_{j}(z)-R_{j}(z) .
$$

Similarly we have

$$
\begin{aligned}
& \left(1-e^{-z / \lambda_{j}}\right) k_{12}(z, j) \\
= & -\frac{1}{\lambda_{j}} e^{\left(1-1 / \lambda_{j}+\bar{\mu}_{j} / \lambda_{j}\right) z} E_{1}\left(\left(1+b+\frac{\bar{\mu}_{j}}{\lambda_{j}}\right) z\right)-d_{j} e^{\left(1-1 / \lambda_{j}+\bar{\mu}_{j} / \lambda_{j}\right) z}+\widetilde{H}_{j}(z),
\end{aligned}
$$

where

$$
\widetilde{H}_{j}(z)=e^{z} \int_{1+b}^{1+b+1 / \lambda_{j}} \frac{\Gamma^{\prime}}{\Gamma}\left(\lambda_{j} s+\bar{\mu}_{j}\right) e^{-s z} d s .
$$

Computation of $k_{13}(z, F)$ is straightforward:

$$
\begin{aligned}
k_{13}(z, F) & =\int_{-b+i \infty}^{-b} \frac{\bar{F}^{\prime}}{\bar{F}}(1-s) e^{s z} d s=-\sum_{n \geq 2} \frac{\bar{\Lambda}_{F}(n)}{n} \int_{-b+i \infty}^{-b} e^{s(z+\log n)} d s \\
& =-e^{-b z} \sum_{n \geq 2} \frac{\bar{\Lambda}_{F}(n)}{n^{1+b}(z+\log n)} .
\end{aligned}
$$

Similarly

$$
k_{2}(z, F)=e^{(1+b) z} \sum_{n \geq 2} \frac{\Lambda_{F}(n)}{n^{1+b}(z-\log n)} .
$$


Gathering (6)-(10) we obtain

$$
k(z, F)=\frac{1}{2 \pi i} \sum_{j=1}^{r} \frac{e^{\left(1-\mu_{j}\right) z / \lambda_{j}}+e^{\left(1+\bar{\mu}_{j} / \lambda_{j}\right) z}}{e^{z / \lambda_{j}}-1} \log z+N_{0}(z, F),
$$

where $N_{0}(z, F)$ is meromorphic on $\mathbb{C}$. This together with (a) easily implies (b).

(c) We consider the function $k^{-}(z, F)$, defined for complex $z$ satisfying $\operatorname{Im} z<0$, by

$$
k^{-}(z, F)=\sum_{\operatorname{Im} \varrho<0} e^{\varrho z} .
$$

The series converges uniformly on every compact subset of the lower half plane and $k^{-}(\cdot, F)$ is holomorphic there. Let

$$
k_{0}^{-}(z, F)=\sum_{\operatorname{Re} \mu_{j}=0, \operatorname{Im} \mu_{j}<0} e^{i \mu_{j} z / \lambda_{j}} .
$$

Then analogously to the proof of (b) we have

$$
2 \pi i\left(k^{-}(z, F)+k_{0}^{-}(z, F)\right)=k_{1}^{-}(z, F)+k_{2}^{-}(z, F)+h^{-}(z, F),
$$

where

$$
\begin{aligned}
& k_{1}^{-}(z, F)=\int_{1+b-i \infty}^{1+b} \frac{F^{\prime}}{F}(s) e^{s z} d s, \\
& k_{2}^{-}(z, F)=\int_{-b}^{-b-i \infty} \frac{F^{\prime}}{F}(s) e^{s z} d s, \\
& h^{-}(z, F)=\int_{\bar{l}} \frac{F^{\prime}}{F}(s) e^{s z} d s,
\end{aligned}
$$

$\bar{l}$ being a curve symmetrical to $l$ in (b).

Term by term integration shows that

$$
k_{1}^{-}(z, F)=-k_{2}(z, F) .
$$

Let

$$
M=\max _{1 \leq j \leq r}\left|\frac{\operatorname{Im} \mu_{j}}{\lambda_{j}}\right|+1
$$

Then by the Cauchy integral theorem,

$$
k_{2}^{-}(z, F)=\left(\int_{-b}^{-b-i M}+\int_{-b-i M}^{-\infty-i M}\right) \frac{F^{\prime}}{F}(s) e^{s z} d s .
$$

The first integral is entire in $z$ while the second is holomorphic for $\operatorname{Re} z>0$. 
Similarly we have

$$
k_{2}^{-}(z, F)=\left(\int_{-\infty+i M}^{-b+i M}+\int_{-b+i M}^{-b}\right) \frac{F^{\prime}}{F}(s) e^{s z} d s .
$$

Hence by the theorem of residues we have, for $\operatorname{Re} z>0$,

$$
-k_{1}(z, F)-k_{2}^{-}(z, F)=2 \pi i \sum_{\operatorname{Re} \omega<0} e^{\omega z}
$$

Similarly

$$
-h^{-}(z, F)-h(z, F)=2 \pi i\left(\sum_{\operatorname{Re} \omega=0} e^{\omega z}+\sum_{\operatorname{Im} \varrho=0} e^{\varrho z}-m_{F} e^{z}\right) .
$$

Gathering (11)-(13) we obtain

$$
k^{-}(z, F)=-k(z, F)+m_{F} e^{z}-k\left(z, \gamma_{F}\right)-\sum_{\operatorname{Im} \varrho=0} e^{\beta z} .
$$

This gives meromorphic continuation of $k^{-}(\cdot, F)$ to the surface $M$. Since $\bar{\varrho}$ is a non-trivial zero of $\bar{F}$ provided $\varrho$ is a non-trivial zero of $F$, we have $k(z, F)=\overline{\sum_{\operatorname{Im} \varrho>0} e^{\bar{\varrho} z^{c}}}=\overline{k^{-}\left(z^{c}, \bar{F}\right)}=-\overline{k\left(z^{c}, \bar{F}\right)}+m_{F} e^{z}-k^{-}\left(z, \gamma_{\bar{F}}\right)-\sum_{\gamma=0} e^{\varrho z}$.

If we notice that $\overline{k\left(z, \gamma_{\bar{F}}\right)}=k\left(z, \gamma_{F}\right)$, the result follows.

(d) Let $0<a<\min (\log 2, x)$ and let $l(a, x)$ denote a smooth curve on the upper half plane joining $a$ and $x$. Then the theorem of residues yields

$$
K(x, F)=K(a, F)+\int_{l(a, x)} k(s, F) d s .
$$

Moreover,

$$
\int_{l(a, x)} k(s, F) d s-\int_{l(a, x)^{c}} k(s, F) d s=-\psi\left(e^{x}, F\right) .
$$

Applying the functional equation (c), we find

$$
\begin{aligned}
& \int_{l(a, x)^{c}} k(s, F) d s=\int_{l(a, x)} k\left(s^{c}, F\right) d s^{c} \\
& =-\int_{l(a, x)} k(s, \bar{F}) d s+m_{F} e^{x}-\int_{1}^{x} k\left(t, \gamma_{F}\right) d t-\sum_{\gamma=0} \frac{e^{\beta x}}{\beta}+c_{F},
\end{aligned}
$$

which gives (4). The proof of (5) is similar.

(e) Let

$$
\Phi(z, F)=e^{-z / 2} K(z, F)+\overline{e^{-z / 2} K(z, \bar{F})} .
$$


Then assuming GRH we have, for $z=x+i y, y>0$,

$$
\Phi(z, F)=\sum_{\gamma \neq 0} e^{-|\gamma| y} \frac{e^{i \gamma x}}{\varrho} .
$$

Moreover, $\lim _{y \rightarrow 0^{+}} \Phi(z, F)=\mathcal{F}(x, F)$ for almost all real $x$.

For $0<y^{\prime}<y$ and any real $x$ we have

$$
\begin{aligned}
\int_{x}^{x+1} \mid & \\
& =\int_{x}^{x+1}\left|\sum_{\gamma \neq 0} \frac{e^{-|\gamma| y}-e^{-|\gamma| y^{\prime}}}{\varrho} e^{i u \gamma}\right|^{2} d u \\
& =\sum_{\gamma \neq 0} \sum_{\gamma^{\prime} \neq 0} \frac{\left(e^{-|\gamma| y}-e^{-|\gamma| y^{\prime}}\right)\left(e^{-\left|\gamma^{\prime}\right| y}-e^{-\left|\gamma^{\prime}\right| y^{\prime}}\right)}{\varrho \varrho^{\prime}} \int_{x}^{x+1} e^{i\left(\gamma-\gamma^{\prime}\right) u} d u \\
& \ll \sum_{\gamma \neq 0} \sum_{\gamma^{\prime} \neq 0} \frac{\left(e^{-|\gamma| y^{\prime}}-e^{-|\gamma| y}\right)\left(e^{-\left|\gamma^{\prime}\right| y^{\prime}}-e^{-\left|\gamma^{\prime}\right| y}\right)}{\left|\varrho \varrho^{\prime}\right|} \min \left(1, \frac{1}{\left|\gamma-\gamma^{\prime}\right|}\right) .
\end{aligned}
$$

Now let $y^{\prime} \rightarrow 0$ :

$$
\begin{aligned}
& \int_{x}^{x+1}|\Phi(u+i y, F)-\mathcal{F}(u, F)|^{2} d u \\
& \ll \sum_{\gamma \neq 0} \sum_{\gamma^{\prime} \neq 0} \frac{\left(1-e^{-|\gamma| y}\right)\left(1-e^{-\left|\gamma^{\prime}\right| y}\right)}{\left|\varrho \varrho^{\prime}\right|} \min \left(1, \frac{1}{\left|\gamma-\gamma^{\prime}\right|}\right) .
\end{aligned}
$$

Since

$$
\sum_{\gamma \neq 0} \sum_{\gamma^{\prime} \neq 0} \frac{1}{\left|\varrho \varrho^{\prime}\right|} \min \left(1, \frac{1}{\left|\gamma-\gamma^{\prime}\right|}\right) \ll 1
$$

the RHS of (14) tends to zero as $y \rightarrow 0^{+}$. Hence $\Phi(u+i y, F) \rightarrow \mathcal{F}(u, F)$ in the sense of $S^{2}$-norm. Since $\Phi(x+i y, F)$ is Bohr almost periodic for every fixed positive $y$ we conclude that $\mathcal{F}(x, F)$ is almost periodic in the $L^{2}$-sense of Stepanov and the theorem is proved.

\section{Auxiliary results from measure theory}

Lemma 1. Let $(X, \mu)$ be a measurable space of finite measure and where $\mu$ is non-negative. Let $f \in L^{1}(X, \mathbb{R})$ and $U$ be an open subset of $\mathbb{R}$. Let $\varepsilon>0$. There exists $\varepsilon^{\prime}$ such that for any $g \in L^{1}(X, \mathbb{R})$ such that $\|f-g\|_{1} \leq \varepsilon^{\prime}$, we have

$$
\mu\left(f^{-1}(U) \backslash g^{-1}(U)\right) \leq \varepsilon
$$


Proof. We have

$$
\bigcup_{\delta>0}\left\{x: d\left(f(x),{ }^{c} U\right) \geq \delta\right\}=f^{-1}(U),
$$

where $d(y, V)$ denotes the distance between the point $y$ and the set $V$. Thus there exists $\delta_{0}>0$ such that $K=\left\{x: d\left(f(x),{ }^{c} U\right) \geq \delta_{0}\right\}$ satisfies $\mu(K) \geq \mu\left(f^{-1}(U)\right)-\varepsilon / 2$. Let $\eta>0$. We have

$$
\mu\{x:|f(x)-g(x)| \geq \eta\} \leq\|f-g\|_{1} / \eta .
$$

Take $\eta=\delta_{0} / 2$ and $\varepsilon^{\prime}=\delta_{0} \varepsilon / 4$. We have

$$
K \backslash\{x:|f(x)-g(x)| \geq \eta\} \subset g^{-1}(U)
$$

while the measure of this set is $\geq \mu\left(f^{-1}(U)\right)-\varepsilon$ as required.

We also need the following simple lemma.

Lemma 2. Let $k$ and $N, k \leq N$, be positive integers and let $\left\{A_{n}\right\}_{n=1}^{N}$ be a family of $\mu$-measurable sets satisfying the following conditions:

1. For every $n_{1}<\ldots<n_{k} \leq N$ we have $A_{n_{1}} \cap \ldots \cap A_{n_{k}}=\emptyset$.

2. $\mu\left(A_{j}\right) \geq \delta(>0)$ for all $j=1, \ldots, N$. Then

$$
\mu\left(\bigcup_{j=1}^{N} A_{j}\right) \geq \delta N / k
$$

Proof. Let $f(x):=\sum_{j=1}^{N} \mathbb{1}_{A_{j}}(x)$. Then $0 \leq f(x) \leq k$ and therefore

$$
k \mu\left(\bigcup_{j=1}^{N} A_{j}\right) \geq \int_{\cup A_{j}} f(x) d \mu(x)=\sum_{j=1}^{N} \int_{\cup A_{j}} \mathbb{1}_{A_{j}}(x) d \mu(x) \geq N \delta
$$

and the lemma follows.

4. Almost periodic functions. We refer to the excellent books by A. S. Besicovitch [1] and H. Bohr [4] as well as to the original papers [17], [15], [2] for the standard definitions and facts on almost periodic functions (see also [3], [6] and [19]). Here we restrict ourselves to some basic remarks in order to fix notation.

For a complex-valued function $f$ defined on the real axis and a real number $\tau$ we write $f_{\tau}(t)=f(t+\tau)$ and $\Gamma_{0}(f)=\left\{f_{\tau}: \tau \in \mathbb{R}\right\}$. Following Bochner, a continuous function $f$ is called almost periodic in the sense of Bohr if the set $\Gamma_{0}(f)$ is relatively compact in the Banach space of bounded continuous functions with the usual norm $\|f\|_{\infty}=\sup _{x \in \mathbb{R}}|F(x)|$. If we replace bounded continuous functions by locally $L^{2}$ integrable ones and the sup-norm by

$$
\|f\|_{S^{2}}:=\max _{x \in \mathbb{R}}\left(\int_{x}^{x+1}|g(t)|^{2} d t\right)^{1 / 2}
$$


we obtain the definition of Stepanov $L^{2}$-almost periodic functions. This space is denoted by $S^{2}$. Finally, taking in $L_{\text {loc }}^{2}(\mathbb{R})$ another norm

$$
\|f\|_{W^{2}}:=\lim _{V \rightarrow \infty} \max _{x \in \mathbb{R}}\left(\frac{1}{V} \int_{x}^{x+V}|g(t)|^{2} d t\right)^{1 / 2},
$$

we recover Weyl $L^{2}$-almost periodic functions, which form a Banach space denoted by $W^{2}$.

For an almost periodic function $f$ (in one of the above senses) we denote by $\Gamma(f)$ the closure of $\Gamma_{0}(f)$ (in the appropriate norm). By definition $\Gamma(f)$ is compact. We further recall that for the three kinds of almost periodic functions we have just defined, classical theorems assert the equivalence for a function $f$ belonging to the proper space between the compactness of $\Gamma(f)$, saying that $f$ is a limit of trigonometric polynomials and the fact that the set of pseudo-periods is large in an appropriate sense.

Lemma 3. Let $f \in S^{2}$ and $g \in \Gamma(f)$. Then $\Gamma(g)=\Gamma(f)$.

Proof. It is enough to prove it is dense. Let $h \in \Gamma(f)$. For $\varepsilon>0$, there exist $\tau_{0}, \tau_{1}$ such that

$$
\left\|h-f_{\tau_{0}}\right\|_{S^{2}} \leq \varepsilon / 2, \quad\left\|g-f_{\tau_{1}}\right\|_{S^{2}} \leq \varepsilon / 2 .
$$

Since $\left\|g-f_{\tau_{1}}\right\|_{S^{2}}=\left\|g_{\tau_{0}-\tau_{1}}-f_{\tau_{0}}\right\|_{S^{2}}$, we get $\left\|g_{\tau_{0}-\tau_{1}}-h\right\|_{S^{2}} \leq \varepsilon$ as required.

Let $f$ be an $S^{2}$ almost periodic function and consider the following distance on $\mathbb{R}$ :

$$
d_{f}\left(\tau_{1}, \tau_{2}\right)=\left\|f_{\tau_{1}}-f_{\tau_{2}}\right\|_{S^{2}}
$$

for which addition is continuous (and translations are isometries). Let $\widehat{\mathbb{R}}(f)$ be the completion of $\mathbb{R}$ for this distance. We can prolong the action of $\mathbb{R}$ on $\Gamma(f)$ to a continuous action of $\widehat{\mathbb{R}}(f)$ on the same space. From this we directly see that $\widehat{\mathbb{R}}(f)$ is a compact abelian group.

Hence it can be endowed with a unique probability Haar measure $\lambda_{f}$. For any continuous function $\varphi: \widehat{\mathbb{R}}(f) \rightarrow \mathbb{C}$, we have

$$
M(\varphi)=\lim _{T \rightarrow \infty} \frac{1}{T} \int_{-T}^{T} \varphi(t) d t=\int_{\widehat{\mathbb{R}}(f)} \varphi(t) d \lambda_{f}(t) .
$$

Lemma 4. Let $K$ be a closed subset of $\widehat{\mathbb{R}}(f)$. Let $\varepsilon>0$. There exists $V_{0}(\varepsilon)>0$ such that for every $V \geq V_{0}(\varepsilon)$ we have

$$
\max _{\tau \in \widehat{\mathbb{R}}(f)} \max _{x \in \mathbb{R}} \frac{1}{V} \operatorname{m}\{[x, x+V] \cap(K+\tau)\} \leq \lambda_{f}(K)+\varepsilon .
$$

Proof. We only handle the case $\tau=0$ since $\lambda_{f}(K+\tau)=\lambda_{f}(K)$. By Urysohn's lemma, there exists $\varphi \in C^{0}(\widehat{\mathbb{R}}(f))$ such that $0 \leq \varphi \leq 1, \varphi_{\mid K}=1$ 
and

$$
\int_{\widehat{\mathbb{R}}(f)} \varphi(t) d \lambda_{f}(t) \leq \lambda_{f}(K)+\varepsilon / 2 .
$$

Since

$$
M(\varphi)=\int_{\widehat{\mathbb{R}}(f)} \varphi(t) d \lambda_{f}(t)
$$

we find that there exists $V_{0}>0$ such that for any $V>V_{0}$ and any $\alpha \in \mathbb{R}$,

$$
\left|\frac{1}{V} \int_{\alpha}^{\alpha+V} \varphi(t) d t-\int_{\widehat{\mathbb{R}}(f)} \varphi(t) d \lambda_{f}(t)\right| \leq \varepsilon / 2 .
$$

The lemma now follows readily.

Lemma 5. Let $f$ be a real-valued $S^{2}$ almost periodic function. Put

$$
\begin{aligned}
\sigma_{f}(\eta, y) & :=\operatorname{m}\{x \in[0,1]: y+\eta \geq f(x) \geq y\}, \\
G_{f}(\eta, y, \varepsilon) & :=\left\{\tau \in \widehat{\mathbb{R}}(f): \sigma_{f}(\eta, y) \geq \varepsilon\right\}, \quad G_{f}(y, \varepsilon)=G_{f}(0, y, \varepsilon) .
\end{aligned}
$$

Then we have:

1. $\lim _{\eta \rightarrow 0^{+}} \lambda_{f}\left(G_{f}(\eta, y, \varepsilon)\right)=\lambda_{f}\left(G_{f}(y, \varepsilon)\right)$.

2. For every $\eta, \varepsilon \geq 0$ the sets $G_{f}(\eta, y, \varepsilon)$ are closed.

3. There exists a countable set $\mathcal{Y}_{f}$ such that for every $\varepsilon>0$ and every $y \notin \mathcal{Y}_{f}$ we have $\lambda_{f}\left(G_{f}(y, \varepsilon)\right)=0$.

Proof. The first assertion follows from the equality

$$
\bigcap_{\eta>0} G_{f}(\eta, y, \varepsilon)=G_{f}(y, \varepsilon)
$$

The second assertion is a consequence of Lemma 1 applied to a closed set. Let $\tau=\lim _{n \rightarrow \infty} \tau_{n}, \tau_{n} \in G_{f}(\eta, y, \varepsilon)$. For any $\varepsilon_{1}>0$, there exists an $N_{1}$ such that for $n \geq N_{1}$, the quantity $\left\|f_{\tau}-f_{\tau_{n}}\right\|_{S^{2}}$ is so small that by Lemma 1 , $\sigma_{f_{\tau}}(\eta, y) \geq \sigma_{f_{\tau_{n}}}(\eta, y)-\varepsilon_{1} \geq \varepsilon-\varepsilon_{1}$. Letting $\varepsilon_{1}$ tend to zero proves that $\tau \in G_{f}(\eta, y, \varepsilon)$ as required.

To prove the last assertion observe that for different $y$ and $y^{\prime}$ the sets $f^{-1}(y)$ and $f^{-1}\left(y^{\prime}\right)$ are disjoint. Hence a $\tau$ can belong to at most $[1 / \varepsilon]+1$ sets $G(y, \varepsilon)$ ( $\varepsilon$ fixed, $y$ varying). Let $y_{1}, \ldots, y_{N}$ be different and such that $\lambda_{f}\left(G\left(y_{j}, \varepsilon\right)\right) \geq \delta, j=1, \ldots, N$. Then by Lemma 2 ,

$$
\varepsilon \delta N \leq \lambda_{f}\left(\bigcup_{j=1}^{N} G\left(y_{j}, \varepsilon\right)\right) \leq \lambda_{f}(\widehat{\mathbb{R}}(f))=1 .
$$

Hence $N \leq 1 /(\varepsilon \delta)$. Consequently, the set $\mathcal{Y}_{F}(\varepsilon, \delta)$ of $y$ satisfying $\lambda_{f}(G(y, \varepsilon))$ $\geq \delta$ is finite. Taking a countable set of $\varepsilon$ tending to zero and a countable set of $\delta$ tending to zero, we get the stated result. 
Lemma 6. Let $w$ be an admissible function.

1. For $T, V \in \mathbb{R}, V>0$, we have

$$
\sum_{k \geq 0} w(T-k V) \leq w(T)+W(T) / V .
$$

2. If $w(T)=o(W(T))$ as $T \rightarrow \infty$ then for every $W^{2}$ almost periodic function $h$ we have

$$
\lim _{T \rightarrow \infty} \frac{1}{W(T)} \int_{-\infty}^{T} h(t) w(t) d t=M(h) .
$$

Proof. The first part follows from the inequality

$$
w(T-k V) \leq \frac{1}{V} \int_{T-k V}^{T-(k-1) V} w(t) d t .
$$

To prove (16) let us approximate $h$ by trigonometric polynomials for which the result is clear by the second mean value theorem for Stieltjes integrals. We bound the error term. Let $\varepsilon>0$. For $r \geq 0, r \in W^{1},\|r\|_{W^{1}}<\varepsilon / 2$, we have, for sufficiently large $V_{0}$ and all $x \in \mathbb{R}$,

$$
\int_{x}^{x+V} r(t) d t \leq \varepsilon V_{0} / 2 .
$$

Moreover, for $T \geq T_{0}(\varepsilon)$ we have $w(T) / W(T) \leq 1 / V_{0}$. Hence by the first part of the lemma we have

$$
\begin{aligned}
\frac{1}{W(T)} \int_{-\infty}^{T}|r(t)| w(t) d t & \leq \frac{1}{W(T)} \sum_{k=0}^{\infty} \int_{T-(k+1) V_{0}}^{T-k V_{0}}|r(t)| w(t) d t \\
& \leq \frac{1}{W(T)} \sum_{k=0}^{\infty} w\left(T-k V_{0}\right) \frac{\varepsilon V_{0}}{2} \leq \frac{\varepsilon V_{0}}{2} \cdot \frac{w(T)}{W(T)}+\varepsilon / 2 \leq \varepsilon
\end{aligned}
$$

and the lemma follows.

Let $h$ be $W^{2}$ almost periodic. We consider the integral transform of $h$ defined by

$$
A(h)(T):=e^{-T} \int_{-\infty}^{T} h(t) e^{t} d t .
$$

Lemma 7. The integral $A(h)$ exists and is finite for all $h \in W^{2}$. Moreover $A$ is a linear and continuous operator from $W^{2}$ to $W^{2}$. 
Proof. Using the Cauchy-Schwarz inequality we have

$$
\begin{aligned}
\left(e^{-T} \int_{-\infty}^{T}|h(t)| e^{t} d t\right)^{2} & \leq e^{-T} \int_{-\infty}^{T}|h(t)|^{2} e^{t} d t \\
& \leq \sum_{k \geq 0} e^{-T} \int_{T-(k+1) V}^{T-k V}|h(t)|^{2} e^{t} d t \\
& \leq \sum_{k \geq 0} e^{-k V} \int_{T-(k+1) V}^{T-k V}|h(t)|^{2} d t
\end{aligned}
$$

and the boundedness follows from the fact that $\|h\|_{W^{2}}<\infty$.

It remains to show that $A$ is linear and continuous. The linearity is obvious. Moreover, we have

$$
\begin{aligned}
\frac{1}{V} \int_{x}^{x+V}|A(h)(t)|^{2} d t & \leq \frac{1}{V} \int_{x}^{x+V}|h(t)|^{2} d t+e^{-x} \frac{1}{V} \int_{-\infty}^{x}|h(t)|^{2} e^{t} d t \\
& \leq \max _{x \in \mathbb{R}}\left(\frac{1}{V} \int_{x}^{x+V}|h(t)|^{2} d t\right)+\max _{x \in \mathbb{R}}\left(e^{-x} \frac{1}{V} \int_{-\infty}^{x}|h(t)|^{2} e^{t} d t\right)
\end{aligned}
$$

and

$$
\begin{aligned}
e^{-x} \frac{1}{V} \int_{x}^{x+V}|h(t)|^{2} e^{t} d t & \leq \frac{1}{V} \sum_{k=0}^{\infty} e^{-k V} \int_{x-(k+1) V}^{x-k V}|h(t)|^{2} d t \\
& \leq \frac{1}{1-e^{-V}} \max _{y \in \mathbb{R}} \frac{1}{V} \int_{y}^{y+V}|h(t)|^{2} d t
\end{aligned}
$$

Letting $V \rightarrow \infty$ we obtain

$$
\|A(h)\|_{W^{2}} \leq \sqrt{2}\|h\|_{W^{2}}
$$

Hence $A$ is continuous from $W^{2}$ to $\left(L_{\text {loc }}^{2}(\mathbb{R}),\|\cdot\|_{W^{2}}\right)$. To prove that $A\left(W^{2}\right) \subset$ $W^{2}$ observe that $A$ commutes with the action of $\mathbb{R}$, i.e. $A(h)_{t}=A\left(h_{t}\right)$ and therefore the family $\left\{A(h)_{t}\right\}_{t \in \mathbb{R}}$ of shifts is relatively compact as a continuous image of the relatively compact family $\left\{h_{t}\right\}_{t \in \mathbb{R}}$.

Lemma 8. Let $h$ be $S^{2}$ almost periodic. Then $\|A(h)\|_{\infty} \leq 1.1\|h\|_{S^{2}}$.

Proof. We have

$$
|A(h)(T)| \leq \int_{-\infty}^{0}\left|h_{T}(t)\right| e^{t} d t \leq\|h\|_{S^{2}} \sum_{k \geq 0} \sqrt{\frac{e^{-2 k}-e^{-2 k-2}}{2}}
$$

and we check that $(e+1) /(2(e-1)) \leq(1.05)^{2}$. 
5. Proof of Theorems 2 and 3. Let $f$ be a real-valued $S^{2}$ almost periodic function. We write

$$
D(\eta, f, y)(t):=\min (1, \max (f(t)-y, 0) / \eta),
$$

which is $S^{2}$ almost periodic and tends pointwise to $\mathbb{1}_{\{f(t)>y\}}$ as $\eta \rightarrow 0^{+}$. Our aim is to show that this convergence holds in the $W^{2}$-sense also, thus showing that $\mathbb{1}_{\{f(t)>y\}}$ is $W^{2}$ almost periodic. Notice that $D(\eta, f, y)(t)$ is 0 if $f(t) \leq y$ and 1 if $f(t) \geq y+\eta$. The quantity

$$
\mathcal{I}:=\frac{1}{V} \int_{x}^{x+V}\left|\mathbb{1}_{\{f>y\}}(t)-D(\eta, f, y)(t)\right|^{2} d t
$$

is not more than

$$
\frac{1}{V} \mathrm{~m}\{t \in[x, x+V]: y+\eta \geq f(t) \geq y\}
$$

and the proof will follow from the estimate

$$
\lim _{\eta \rightarrow 0^{+}} \limsup _{V \rightarrow+\infty} \max _{x \in \mathbb{R}} \frac{1}{V} \mathrm{~m}\{t \in[x, x+V]: y+\eta \geq f(t) \geq y\}=0
$$

which we have to establish.

Let $\varepsilon$ be positive and let $y \notin \mathcal{Y}_{f}$. By Lemma 5 we have $\lambda_{f}(G(y, \varepsilon / 4))=0$ and therefore for all sufficiently small positive $\eta$ we have $\lambda_{f}(G(\eta, y, \varepsilon / 4))<$ $\varepsilon / 4$.

We have

$$
\mathcal{I} \leq \frac{1}{V} \int_{x-1}^{x+V} \sigma\left(\eta, f_{t}, y\right) d t
$$

since we readily check the general inequality

$$
\int_{x}^{x+V} g(w) d w \leq \int_{x-1}^{x+V} \int_{0}^{1} g_{t}(v) d v d t \quad(g \geq 0) .
$$

We thus get using Lemma 4 with $K=G(\eta, y, \varepsilon / 4)$ that $\mathcal{I}$ is not more than

$$
\begin{aligned}
\frac{1}{V} \mathrm{~m}\left\{t \in[x-1, x+V]: \sigma_{f_{t}}(\eta, y) \geq\right. & \varepsilon / 4\}+\frac{V+1}{V} \cdot \frac{\varepsilon}{4} \\
& \leq \frac{V+1}{V}\left(\lambda_{f}(K)+\varepsilon / 4+\varepsilon / 4\right)<\varepsilon
\end{aligned}
$$

for $V \geq V_{0}(\varepsilon)>3$. Consequently, we have proved that $\mathbb{1}_{\{f>y\}}$ is $W^{2}$ almost periodic.

Now it is straightforward to finish the proof of Theorem 2. Indeed, using the operator $A$ defined by (17) we have

$$
\nabla_{y}(f, \cdot)=A\left(\mathbb{1}_{\{f>y\}}\right) \in W^{2}
$$

and the result follows by Lemma 7 . 
Moreover, it is obvious that if in addition $h$ is bounded then $A(h)$ is 1-Lipschitz.

Finally, observe that Theorem 3 is an immediate consequence of the fact that $\mathbb{1}_{\{\phi>y\}}, y \notin \mathcal{Y}_{\phi}$, is $W^{2}$ almost periodic, and of the second part of Lemma 6. In addition we see that $\delta_{y}$ is independent of $w$ and equals $M\left(\mathbb{1}_{\{\phi>y\}}\right)$.

6. Proof of Theorem 4. Let $U$ be an open subset of $\mathbb{R}$. We apply Lemma 1 for $X=(-\infty, T]$ and the measure $d \mu=e^{t-T} d t$ to obtain

$$
\min _{g:\|f-g\|_{1} \leq \varepsilon^{\prime}} e^{-T} \int_{-\infty}^{T} \mathbb{1}_{\{g(t) \in U\}} e^{t} d t \geq e^{-T} \int_{-\infty}^{T} \mathbb{1}_{\{f(t) \in U\}} e^{t} d t-\varepsilon,
$$

where the $L^{1}$-norm is taken with respect to $\mu$, for an $\varepsilon^{\prime}>0$ small enough in terms of $\varepsilon>0, f$ and $U$. Note that by Lemma 3, inequality (18) restricted to $S^{2}$ almost periodic functions tells us that the function

$$
\Lambda_{T, U}: \Gamma(f) \rightarrow \mathbb{R}, \quad g \mapsto e^{-T} \int_{(-\infty, T] \cap g^{-1}(U)} e^{t} d t
$$

is upper semicontinuous since the $L^{1}(\mu)$-norm is seen to be bounded by $1.1\|\cdot\|_{S^{2}}$ by Lemma 8 . On taking $T$ to be 0 , this proves the first part of Theorem 4.

If $U$ were a closed set the inequality (18) would be reversed and the min replaced by max, which shows that we cannot replace $U$ by any borelian set in this statement without proving the functional above to be continuous. This has the consequence that $\nabla_{y, f}(\cdot)$ is continuous at every point $\tau_{0} \in \widehat{\mathbb{R}}(f)$ for which

$$
\int_{-\infty}^{0} \mathbb{1}_{\left\{f_{\tau_{0}}(t)=y\right\}} e^{t} d t=0 .
$$

Hence we deduce easily that if the set of $t \in \mathbb{R}$ satisfying

$$
f_{\tau_{0}}(t)=y
$$

has the Lebesgue measure zero then $\nabla_{y, f}(\cdot)$ is continuous at all points of $\tau_{0}+\mathbb{R}$.

Let $f=\phi \in \mathcal{X}_{\mathcal{S}}$ and $\tau_{0}=0$. We can write

$$
\phi(x)=\sum_{j=1}^{N} c_{j} \mathcal{F}\left(x, F_{j}\right)
$$

for some functions $F_{j} \in \mathcal{S}$ and complex numbers $c_{j}, j=1, \ldots, N$.

Suppose that for some $y$ the set of solutions of (19) has a positive Lebesgue measure. Then also its intersection with $\left(\log n_{0}, \log \left(n_{0}+1\right)\right)$ or 
$\left(-\log \left(n_{0}+1\right),-\log n_{0}\right)$ for some positive integer $n_{0}$ has a positive measure. Suppose the former possibility occurs. By Theorem 1(d), for $x>0$ we have

$$
\phi(x)=-e^{-x / 2} \sum_{j=1}^{N} c_{j} \psi\left(e^{x}, F_{j}\right)+H_{+}(x),
$$

where $H_{+}$is holomorphic on $(0, \infty)$. Since in the interval $\left(\log n_{0}, \log \left(n_{0}+1\right)\right)$ the sum is constant, equal to $\alpha_{0}$, say, we see that the set of solutions $x$ of the equation

$$
H_{+}(x)=y+\alpha_{0} e^{-x / 2} \quad(x>0)
$$

has a positive measure. Hence $H_{+}(x)=y+\alpha_{0} e^{-x / 2}$ identically on $(0, \infty)$. This of course determines $y$ uniquely. Another possible exceptional value of $y$ occurs when considering negative $x$. Theorem 4 is therefore proved.

To get the uniform version stated after Theorem 4 we need two ingredients. First notice that the functions $\nabla_{y+\delta, \phi_{v}}(t)$ on $I$ are continuous, that this sequence is non-increasing in $\delta>0$ and that its limit on $\delta \rightarrow 0$ is the continuous function $\nabla_{y, \phi_{v}}(t)$ by our special choice of $\phi$ and $v$. Dini's lemma asserts that the convergence is then uniform on $I$. A similar argument holds for $\nabla_{y-\delta, \phi_{v}}(t)$. The second ingredient is hidden in the proof of Lemmas 1 and 8; we proved there that

$$
\nabla_{y+\delta, \phi_{v}}(t) \leq \nabla_{y, \phi_{v^{\prime}}}(t)+1.1\left\|\phi_{v}-\phi_{v^{\prime}}\right\|_{S^{2}} / \delta \quad(\delta>0) .
$$

We can now embark on the proof. Take $v \in \mathbb{R}$, a bounded interval $I$ and a positive $\varepsilon$. We can find $\delta_{0}=\delta_{0}(v, I, \varepsilon)>0$ such that for all $\delta \leq \delta_{0}$ we have

$$
\max _{t \in I}\left(\left|\nabla_{y+\delta, \phi_{v}}(t)-\nabla_{y, \phi_{v}}(t)\right|+\left|\nabla_{y-\delta, \phi_{v}}(t)-\nabla_{y, \phi_{v}}(t)\right|\right)<\varepsilon / 2 .
$$

Put $\alpha_{0}=\varepsilon \delta_{0} / 4.4$. For any $v^{\prime} \in \mathbb{R}$ satisfying $\left\|\phi_{v}-\phi_{v^{\prime}}\right\| \leq \alpha_{0}$, we have

$$
\begin{aligned}
& \left|\nabla_{y, \phi_{v^{\prime}}}(t)-\nabla_{y, \phi_{v}}(t)\right| \\
& \quad \leq\left|\nabla_{y+\delta_{0}, \phi_{v}}(t)-\nabla_{y, \phi_{v}}(t)\right|+\left|\nabla_{y-\delta_{0}, \phi_{v}}(t)-\nabla_{y, \phi_{v}}(t)\right|+2.2 \alpha_{0} / \delta_{0} \leq \varepsilon,
\end{aligned}
$$

which concludes the proof.

\section{References}

[1] A. S. Besicovitch, Almost Periodic Functions, Dover, 1954.

[2] S. Bochner, Beiträge zur Theorie der fastperiodischen Funktionen. I. Teil: Funktionen einer Variabel, Math. Ann. 96 (1927), 119-147.

[3] S. Bochner and B. Jessen, Distribution functions and positive definite functions, Ann. of Math. 35 (1934), 252-257.

[4] H. Bohr, Almost Periodic Functions, Chelsea, New York, 1951.

[5] C. Breuil, B. Conrad, F. Diamond and R. Taylor, On the modularity of elliptic curves over Q: wild 3-adic exercises, preprint, 1999, www. math.harvard.edu/rtaylor/st.ps.

[6] J. Favard, Leçons sur les fonctions presque-périodiques, Gauthier-Villars, 1933. 
[7] B. Jessen, A note on distribution functions, J. London Math. Soc. 8 (1933), 247-250.

[8] J. Kaczorowski, The k-functions in multiplicative number theory, I. On complex explicit formulae, Acta Arith. 56 (1990), 195-211.

[9] - The k-functions in multiplicative number theory, IV. On a method of A. E. Ingham, ibid. 57 (1991), 231-244.

[10] -, On the distribution of primes modulo 4, Analysis 15 (1995), 159-171.

[11] —, Boundary values of Dirichlet series and the distribution of primes, in: Proc. European Congress of Mathematics (Budapest, 1996), Progr. Math. 168, Birkhäuser, 1998, 237-254.

[12] J. Kaczorowski and A. Perelli, The Selberg class: a survey, in: Number Theory in Progress, K. Győry et al. (eds.), de Gruyter, 1999, 953-992.

[13] M. Rubinstein and P. Sarnak, Chebyshev's bias, Experiment. Math. 3 (1994), 173197.

[14] A. Selberg, Old and new conjectures and results about a class of Dirichlet series, in: Proc. Amalfi Conference on Analytic Number Theory (Maiori, 1989), E. Bombieri et al. (eds.), Università di Salerno, Salerno, 1992, 367-385; Collected Papers, Vol. II, Springer, Berlin, 1991, 47-63.

[15] W. Stepanoff [W. Stepanov], Über einige Verallgemeinerungen der fastperiodischen Funktionen, Math. Ann. 95 (1926), 473-498.

[16] R. Taylor and A. Wiles, Ring-theoretic properties of certain Hecke algebras, Ann. of Math. (2) 141 (1995), 553-572.

[17] H. Weyl, Integralgleichung und fastperiodische Funktionen, Math. Ann. 97 (1926), 338-356.

[18] A. Wiles, Modular elliptic curves and Fermat's last theorem, Ann. of Math. (2) 141 (1995), 443-551.

[19] A. Wintner, Upon a statistical method in the theory of diophantine approximation, Amer. J. Math. 55 (1933), 309-331.

Faculty of Mathematics and Computer Science

A. Mickiewicz University

Umultowska 87

61-614 Poznań, Poland

E-mail: kjerzy@math.amu.edu.pl
Département de Mathématiques

Université de Lille I

59000 Villeneuve d'Ascq, France

E-mail: ramare@agat.univ-lille1.fr 